\title{
atividade Microbiana do Solo após Aplicação de Herbicidas em Sistemas de Plantio Direto e Convencional ${ }^{1}$
}

\author{
Microbial Activity in Soil After Herbicide Application under No-Tillage and Conventional \\ Planting Systems
}

SANTOS, J.B. ${ }^{2}$, JAKELAITIS, A. ${ }^{2}$, SILVA, A.A. ${ }^{3}$, VIVIAN, R. ${ }^{4}$, COSTA, M.D. ${ }^{5}$ e SILVA, A.F. ${ }^{6}$

\begin{abstract}
RESUMO - Avaliaram-se neste trabalho os efeitos de cinco concentrações $(0,0 ; 1,05 ; 2,10$; 4,20; e 8,40 $\mu \mathrm{g} \mathrm{g}^{-1}$ ) dos herbicidas fluazifop-p-butil e fomesafen e da mistura comercial destes (Robust $($ ) sobre a atividade microbiana de um solo submetido aos sistemas de plantio direto (SPD) e convencional (SPC). Amostras do solo coletadas em SPD e SPC foram colocadas em erlenmeyer de $250 \mathrm{~mL}$, tratadas com as diferentes concentrações dos herbicidas e umedecidas próximo à capacidade de campo. A cada intervalo de quatro dias fez-se a quantificação da respiração microbiana, por meio da captura do $\mathrm{CO}_{2}$ liberado do solo. Aos 20 dias após o início da incubação determinou-se o carbono da biomassa microbiana (CBM) e o quociente metabólico $\left(\mathrm{qCO}_{2}\right)$. No solo proveniente do SPD, a atividade microbiana aumentou com as concentrações do fluazifop-p-butil e reduziu para concentrações superiores a 4,20 $\mu \mathrm{g} \mathrm{g}^{-1}$ de fomesafen. Os efeitos dos herbicidas sobre a respiração microbiana e o CBM do solo em SPC foram inferiores aos observados no solo do SPD. Entre os herbicidas, com o aumento das concentrações, verificou-se maior CBM para fluazifop-p-butil e menores valores de CBM para o fomesafen. A mistura comercial dos herbicidas (Robust $\circledast$ ) não influenciou o CBM no SPD, mas sua redução foi maior no solo sob SPC quando aplicado nas maiores concentrações. No SPD, o qCO ${ }_{2}$ aumentou com as concentrações dos herbicidas sem, contudo, variar entre eles. No SPC, a mistura dos herbicidas proporcionou o maior $\mathrm{qCO}_{2}$. Em geral, os herbicidas promoveram efeito negativo sobre a biomassa microbiana. Esse efeito foi proporcional às concentrações aplicadas, sendo menor em solo cultivado no SPD, em comparação aquele cultivado no SPC.
\end{abstract}

Palavras-chave: biomassa microbiana, fluazifop-p-butil, fomesafen, quociente metabólico.

\begin{abstract}
This study evaluated the effect of the herbicides fluazifop-p-butyl and fomesafen in five concentrations $\left(0.0 ; 1.05 ; 2.10 ; 4.20\right.$ and $\left.8.40 \mu \mathrm{g} \mathrm{g}^{-1}\right)$ and their mixture (Robust $(\mathbb{R})$ on microbial activity in soil submitted to no-tillage system (NTS) and conventional planting system (CPS). Samples in Erlenmeyer of $250 \mathrm{~mL}$ were treated with herbicide and wetted to field capacity. After every four days, microbial respiration was quantified through $\mathrm{CO}_{2}$ left by the soil. Metabolic quotient $\left(\mathrm{qCO}_{2}\right)$ and carbon of microbial biomass (CMB) were determined 20 days after incubation. Under NTS, microbial activity increased with fluazifop-p-butyl and decreased with up to $4.20 \mathrm{\mu g} \mathrm{g}^{-1}$ of fomesafen. Herbicide effect on soil microbial respiration and CMB under CPS was below that observed under NTS.CMB increased with the increase of fluazifop-p-butyl concentration and decrease of fomesafen concentration. The mixture (Robust $\left.{ }^{\circledR}\right)$ did not influence CMB under NTS, but it was reduced under CPS in higher concentrations. Under NTS, $\mathrm{qCO}_{2}$ increased with the herbicide concentrations, without, however, varying between them. Under CPS, herbicide mixture provided a higher $\mathrm{qCO}_{2}$. In general, the herbicides provided a negative effect on microbial biomass, proportional to the concentrations applied, being smaller in soil under NTS than in soil under CPS.
\end{abstract}

Key words: microbial biomass, fluazifop-butyl, fomesafen, and metabolic quotient.

Recebido para publicação em 4/4/2005 e na forma revisada em 25/11/2005.

Bolsista Pós-Doutor do Departamento de Fitotecnia da Universidade Federal de Viçosa - UFV, 36570-000 Viçosa-MG, <jbarbosasantos@yahoo.com.br >; ${ }^{3}$ Professor do Departamento de Fitotecnia da UFV; ${ }^{4}$ Estudante de Mestrado do Departamento de Fitotecnia da UFV; ${ }^{5}$ Prof. do Departamento de Microbiologia da UFV; ${ }^{6}$ Acadêmico do curso de Agronomia da UFV.

Planta Daninha, Viçosa-MG, v. 23, n. 4, p. 683-691, 2005 


\section{INTRODUÇÃO}

A biomassa microbiana (BM) é responsável pelo controle de funções essenciais no solo, como decomposição e acúmulo de matéria orgânica, ou transformações envolvendo nutrientes minerais ou compostos no solo. Em razão disso, alto conteúdo de BM pode proporcionar ao solo maior estocagem de nutrientes, possibilitando também melhor ciclagem destes ao longo do tempo (Stenberg, 1999). Ademais, o fato de muitos microrganismos utilizarem a fração disponivel da matéria orgânica os faz sensiveis na mudança em sua qualidade. A $\mathrm{BM}$, a qual possui, comparativamente, taxa de formação e decomposição rápida de um a dois anos (Jenkinson \& Ladd, 1981), tem sido proposta como medida mais sensivel de aumento ou decréscimo na quantidade total da matéria orgânica do solo (Carter, 1992; Sparling, 1992; Balota et al., 2003). Segundo Sparling (1992), mudanças graduais e pequenas na matéria orgânica do solo são difíceis de serem monitoradas e detectadas no curto prazo. Todavia, mudanças significativas na BM podem ser detectadas muito antes que alterações na matéria orgânica possam ser percebidas, possibilitando a adoção de medidas de correção antes que a perda da qualidade do solo seja mais severa.

No sistema plantio direto (SPD), tem-se observado aumento dos teores de matéria orgânica no solo (Balota et al., 2003; FEBRAPDP, 2005). A maior retenção de compostos orgânicos no solo sob SPD tem sido associada ao aumento da agregação das partículas do solo (Beare et al., 1995), as quais protegem fisicamente a matéria orgânica, por formarem barreira que isola os microrganismos do substrato, influindo, também, na ciclagem da BM, pois diminuem as variações de temperatura e umidade no solo ao longo do dia (Elliott \& Coleman, 1988).

Por meio da BM é possivel avaliar as mudanças iniciais no conteúdo de matéria orgânica no solo, pois esta constitui o estágio inicial do carbono dos resíduos em decomposição no solo, definida como a parte viva da matéria orgânica do solo, excetuando raízes e grandes animais, sensivel a mudanças quando se utilizam práticas agrícolas diversas, como fertilizações ou aplicação de pesticidas, entre eles os herbicidas (Wardle \& Parkinson, 1991; Kinney et al., 2005).

Dentre os herbicidas recomendados para as culturas de soja e feijão, destaca-se o fomesafen, indicado no controle de diversas plantas daninhas dicotiledôneas. Entretanto, esse herbicida apresenta longa persistência no solo, com meia-vida de dois a seis meses (Rodrigues \& Almeida, 1998). Normalmente o fomesafen é utilizado em mistura com o fluazifop-p-butil, tendo em vista o controle simultâneo de plantas daninhas dicotiledôneas e gramíneas (Silva et al., 2003).

Apesar dos estudos demonstrando que herbicidas afetam de maneira negativa a biomassa microbiana (Edwards, 1989; Wardle, 1994; Perschbacher et al., 1997; Kinney et al., 2005), poucas informações existem a respeito desse assunto em condições de clima e solos tropicais ou em sistema de plantio direto.

Neste trabalho foram avaliados os efeitos de concentrações dos herbicidas fluazifop-pbutil e fomesafen e da mistura comercial destes (denominada Robust $\AA$ ) sobre a atividade microbiana do solo cultivado em SPD e SPC.

\section{MATERIAL E MÉTODOS}

O experimento foi realizado no Laboratório de Herbicidas no Solo da Universidade Federal de Viçosa, utilizando-se amostras de solo coletadas na profundidade de $0-10 \mathrm{~cm}$, em áreas de cultivo rotacionado de milho com feijão, nos sistemas de plantio direto (SPD) e plantio convencional (SPC). A caracterização química e textural do solo se encontra na Tabela 1. Os tratamentos foram compostos por três herbicidas, cinco concentrações e cinco épocas de avaliação para cada um dos sistemas de cultivo, com três repetições. Os herbicidas fluazifop-p-butil e fomesafen e a mistura de fluazifop-p-butil (200 $\left.\mathrm{g} \mathrm{L}^{-1}\right)$ + fomesafen (250 $\mathrm{g} \mathrm{L}^{-1}$ ) (Robust ${ }^{\circledR}$ ) foram aplicados nas concentrações de 0,$00 ; 1,05 ; 2,10 ; 4,20$; e $8,40 \mu \mathrm{g} \mathrm{g}^{-1}$, equivalentes às doses de 0,125 , 250, 500 e $1.000 \mathrm{~g} \mathrm{ha}^{-1}$ do ingrediente ativo, respectivamente. As cinco épocas de avaliação foram aos 4, 8, 12, 16 e 20 dias após início da incubação. A concentração adotada na mistura comercial baseou-se na mesma para o fomesafen, quando isolado. As amostras de solo foram peneiradas em malha de 2 mesh, 
secas ao ar e tiveram o teor de água determinado. Subamostras de $80 \mathrm{~g}$ foram pesadas e colocadas em frascos erlenmeyer, onde receberam os herbicidas por meio de pulverização, utilizando-se uma microsseringa. A umidade das amostras incubadas foi mantida em $80 \%$ da capacidade de campo. A temperatura ambiente foi monitorada ao longo do experimento, variando entre 23 e $25{ }^{\circ} \mathrm{C}$. Em cada época de avaliação estimou-se a respiração microbiana do solo, por meio da quantidade de $\mathrm{CO}_{2}$ evoluído, o qual foi capturado em frascos contendo $100 \mathrm{~mL}$ de $\mathrm{NaOH}\left(0,25 \mathrm{~mol} \mathrm{~L}^{-1}\right)$, em sistema contínuo de fluxo de ar (isento de $\mathrm{CO}_{2}$ e umidade, por meio da pré-filtração, e passagem do ar em colunas contendo micropérolas de $\mathrm{NaOH}$ ). Procedeu-se então à titulação indireta do hidróxido de sódio com $\mathrm{HCl}\left(0,25 \mathrm{~mol} \mathrm{~L}^{-1}\right)$; o excesso de $\mathrm{NaOH}$ que não reagiu com o $\mathrm{CO}_{2}$ evoluído foi quantificado. Após a última avaliação, aos 20 dias de incubação, determinou-se o carbono da biomassa microbiana (CBM) pelo método descrito por Vance et al. (1987), utilizando-se, em lugar do clorofórmio (fumigação), forno de microondas (irradiação) (Islam \& Weil, 1998).

O cálculo do $\mathrm{qCO}_{2}$ foi feito por meio da relação entre o $\mathrm{CO}_{2}$ acumulado ao longo de 20 dias $\left(\mu \mathrm{g} \mathrm{g}^{-1}\right)$ e o C total da biomassa microbiana $\left(\mu \mathrm{g} \mathrm{g}^{-1}\right)$. Os dados foram submetidos à análise de variância pelo teste $\mathrm{F}$, e as médias dos tratamentos para CBM e $\mathrm{qCO}_{2}$ foram comparadas pelo teste de Tukey a $5 \%$ de probabilidade. Também foram ajustadas curvas de regressão para a evolução do $\mathrm{CO}_{2}$ acumulado durante o período de incubação, para cada herbicida.

\section{RESULTADOS E DISCUSSÃO}

Independentemente do sistema de cultivo ou dos herbicidas avaliados, a resposta em evolução de $\mathrm{CO}_{2}$ comportou-se linearmente em todas as concentrações testadas (Figuras $1 \mathrm{e}$ 2 e Tabela 2).

No SPD, o maior acúmulo de $\mathrm{CO}_{2}$ foi observado quando o solo foi tratado com $8,40 \mu \mathrm{g} \mathrm{g}^{-1}$ de fluazifop-p-butil e $2,10 \mu \mathrm{g} \mathrm{g}^{-1}$ de fomesafen. Na mistura comercial, a respiração foi intermediária à observada para cada herbicida isolado (Figura 1). Os resultados indicaram que para o fluazifop-p-butil a atividade microbiana deste solo aumentou com a concentração, enquanto para o fomesafen esta atividade começou a ser inibida a partir da concentração de $2,10 \mu \mathrm{g} \mathrm{g}^{-1}$ desse herbicida (Figura 1).

Tabela 1 - Composição química e textural da camada de 0-10 $\mathrm{cm}$ de profundidade do solo argiloso proveniente dos sistemas de plantio direto (SPD) e convencional (SPC) utilizado no experimento. Viçosa-MG, 2005

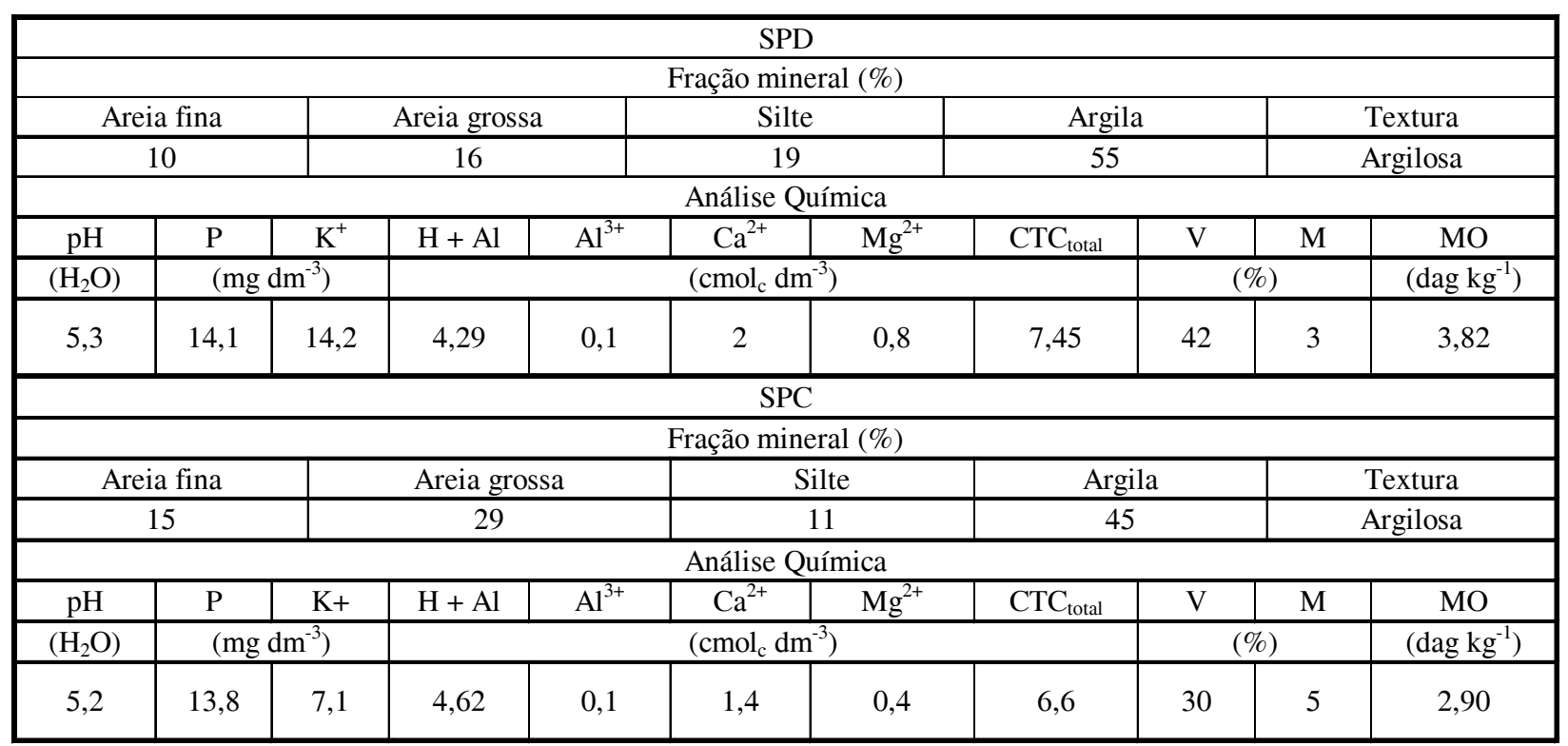

* Análises realizadas nos Laboratórios de Análises Físicas e Químicas de Solo do Departamento de Solos da UFV, segundo a metodologia descrita pela Empresa Brasileira de Pesquisa Agropecuária-EMBRAPA (1997). 

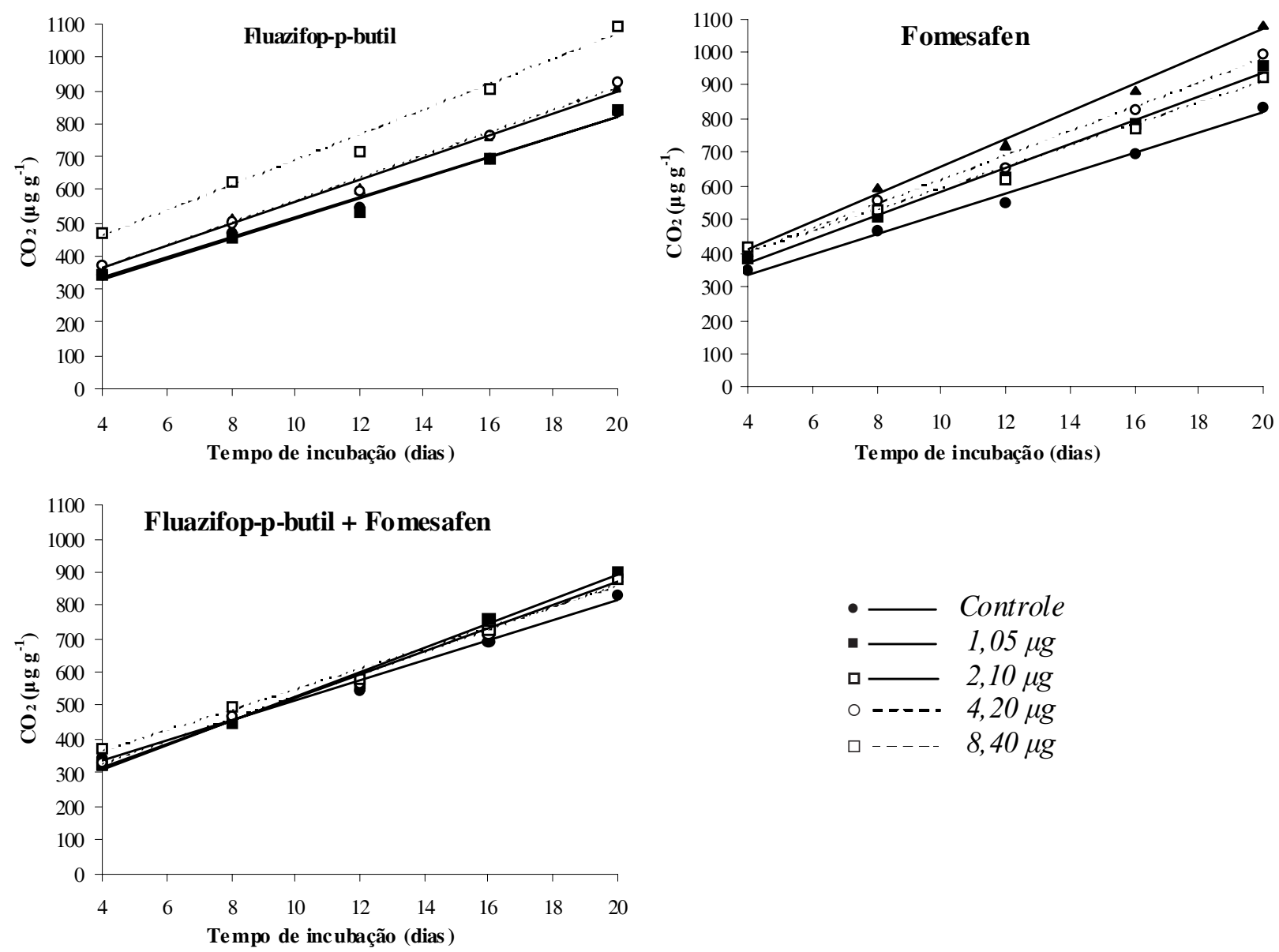

Figura 1 - Evolução de $\mathrm{CO}_{2}$ acumulado ao longo de 20 dias em solo proveniente de sistema de plantio direto que recebeu diferentes concentrações $\left(\mu \mathrm{g} \mathrm{g}^{-1}\right)$ dos herbicidas fluazifop-p-butil e fomesafen e da mistura fluazifop-p-butil + fomesafen (Robust $\left.{ }^{\circledR}\right)$.

Para o SPC, verificou-se menor estímulo dos herbicidas sobre a atividade microbiana em virtude do maior desprendimento de $\mathrm{CO}_{2}$ com o aumento da concentração dos produtos aplicada ao solo (Figura 2). No entanto, independentemente do tratamento herbicida, o total de $\mathrm{CO}_{2}$ acumulado nesse tipo de solo foi nitidamente inferior ao do solo proveniente do SPD, uma vez que na ausência de herbicidas o total evoluído de $\mathrm{CO}_{2}$, ao longo dos 20 dias, subiu de 35 para $80 \mathrm{mg}$ de $\mathrm{CO}_{2}$ por $100 \mathrm{~g}$ de solo, do SPC para o SPD, respectivamente. Esse fato evidenciou atividade microbiana superior no solo cultivado no SPD, o que pode ser atribuído ao não-revolvimento do solo e à manutenção de uma camada de palha na sua superfície. Diversos trabalhos já confirmaram a maior atividade microbiana no SPD, comparado ao SPC (Amato \& Ladd, 1992; Carter,
1992; Balota et al., 2003; Fontes et al., 2004; FEBRAPDP, 2005).

Em trabalho realizado para verificar o efeito de herbicidas sobre a comunidade microbiana aquática, Perschbacher et al. (1997) constataram que fomesafen e fluazifop-p-butil não interferiram na taxa de evolução de $\mathrm{O}_{2}$. Todavia, outros trabalhos mostraram que vários herbicidas, entre eles o glyphosate, afetaram a qualidade do solo pelo efeito indireto sobre a respiração (Souza et al., 1999; Tuffi Santos et al., 2005), na biomassa da microbiota e na ciclagem de nutrientes (Edwards, 1989).

O C microbiano ou C da biomassa microbiana (CBM) estimado ao final do período de incubação do solo, nos diferentes tratamentos, variou entre os herbicidas e entre as concentrações testadas (Tabelas 2 e 3 e Figura 3). 

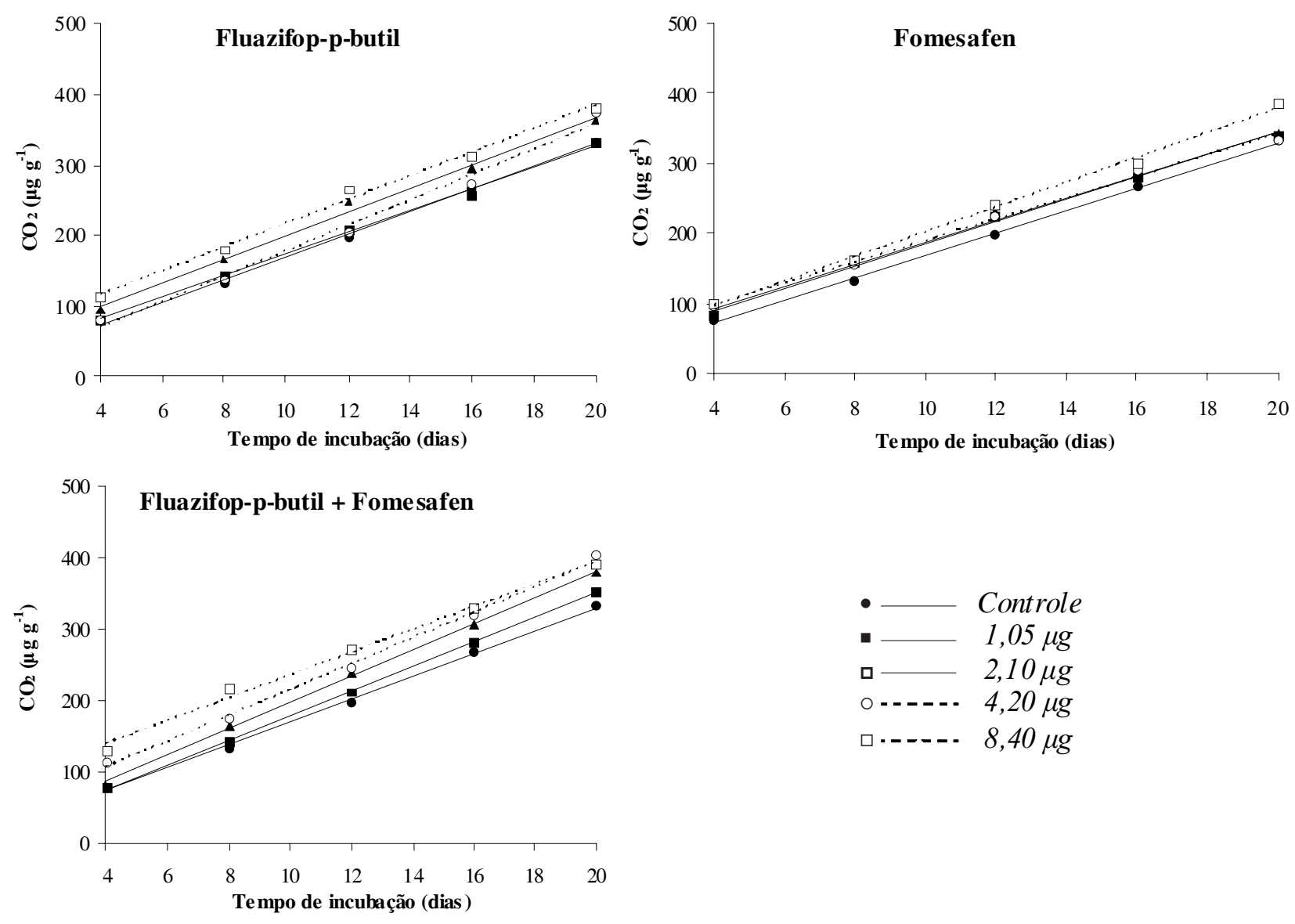

Figura 2 - Evolução de $\mathrm{CO}_{2}$ acumulado ao longo de 20 dias em solo proveniente de sistema de plantio convencional que recebeu diferentes concentrações $\left(\mu \mathrm{g} \mathrm{g}^{-1}\right)$ dos herbicidas fluazifop-p-butil e fomesafen e da mistura fluazifop-p-butil + fomesafen (Robust ${ }^{\circledR}$ ).

Nas concentrações de 2,10 e $4,20 \mu \mathrm{g} \mathrm{g}^{-1} \mathrm{de}$ herbicida aplicado no solo sob SPD, menor CBM foi observado na presença do fluazifop-p-butil, comparado ao fomesafen. Entretanto, na maior concentração testada $\left(8,40 \mu \mathrm{g} \mathrm{g}^{-1}\right)$ verificou-se efeito contrário, isto é, maior redução do CBM na presença do fomesafen (Tabela 3). A mistura comercial dos herbicidas, nas mesmas concentrações, provocou comportamento intermediário no valor de CBM, comparado a cada herbicida isoladamente (Tabela 3 ).

Esses resultados sugerem ser o fomesafen inibidor da atividade microbiana do solo. Outros trabalhos demonstraram que esse herbicida foi extremamente tóxico a bactérias do gênero Bradyrhizobium (Procópio et al., 2004; Santos, 2004). Uma possivel causa da agressividade do fomesafen aos microrganismos pode ser atribuída ao seu mecanismo de ação, o qual é inibidor da enzima protoporfirinogênio oxidase (protox), fazendo com que haja acúmulo de protoporfirina em células tratadas com esse herbicida, ocasionando interação com o oxigênio para produção de formas reativas e, conseqüentemente, peroxidação dos lipídeos e morte celular (Warren \& Hess, 1995).

No SPC, os valores de CBM, similarmente ao ocorrido para a respiração, foram menores aos observados para o solo proveniente do SPD (Tabela 3). Entre os herbicidas, a mistura comercial (Robust@) provocou as maiores reduções nos valores de CBM, principalmente na maior concentração testada. Neste tratamento verificou-se que o total de $\mathrm{C}$ proveniente da biomassa microbiana representou somente $26 \%$ do valor observado na ausência de herbicida (Tabela 3). Esse resultado pode estar relacionado à maior instabilidade do solo no 
Tabela 2 - Equações de regressão correlacionando o tempo de incubação, em dias ( $\left.\mathrm{D}^{* *}\right)$, do solo submetido aos sistemas de plantio direto e convencional, e a evolução de $\mathrm{CO}_{2}\left(\mu \mathrm{g} \mathrm{g}^{-1}\right)$ sob efeito dos herbicidas fluazifop-p-butil e fomesafen e da mistura comercial destes (Robust ${ }^{\circledR}$ ), aplicados em diferentes concentrações ( $\mu \mathrm{g} \mathrm{g}^{-1}$ ). Viçosa-MG, 2005

\begin{tabular}{|c|c|c|c|c|}
\hline \multirow{2}{*}{$\begin{array}{c}\text { Concentração } \\
\left(\mu \mathrm{g} \mathrm{g}^{-1}\right)\end{array}$} & \multicolumn{2}{|c|}{ Sistema plantio direto } & \multicolumn{2}{|c|}{ Sistema plantio convencional } \\
\hline & Equação ajustada & $\mathrm{R}^{2}$ & Equação ajustada & $\mathrm{R}^{2}$ \\
\hline & \multicolumn{4}{|c|}{ Fluazifop-p-butil } \\
\hline 0,00 & $\hat{\mathrm{Y}}=30,0924 \mathrm{D}+216,964$ & 0,93 & $\hat{\mathrm{Y}}=16,0272 \mathrm{D}+9,740$ & 0,95 \\
\hline 1,05 & $\hat{Y}=33,7448 D+225,844$ & 0,85 & $\hat{\mathrm{Y}}=15,388 \mathrm{D}+19,633$ & 0,99 \\
\hline 2,10 & $\hat{\mathrm{Y}}=30,9805 \mathrm{D}+203,214$ & 0,79 & $\hat{\mathrm{Y}}=16,6581 \mathrm{D}+33,229$ & 0,98 \\
\hline 4,20 & $\hat{Y}=34,1172 D+224,698$ & 0,82 & $\hat{\mathrm{Y}}=17,9322 \mathrm{D}+1,432$ & 0,96 \\
\hline \multirow[t]{2}{*}{8,40} & $\hat{\mathrm{Y}}=38,1634 \mathrm{D}+307,055$ & 0,98 & $\hat{\mathrm{Y}}=16,7010 \mathrm{D}+49,557$ & 0,97 \\
\hline & \multicolumn{4}{|c|}{ Fomesafen } \\
\hline 0,00 & $\hat{\mathrm{Y}}=30,0924 \mathrm{D}+216,964$ & 0,93 & $\hat{\mathrm{Y}}=16,0272 \mathrm{D}+9,740$ & 0,95 \\
\hline 1,05 & $\hat{Y}=35,6927 D+226,417$ & 0,98 & $\hat{\mathrm{Y}}=15,9339 \mathrm{D}+25,638$ & 0,94 \\
\hline 2,10 & $\hat{\mathrm{Y}}=41,0423 \mathrm{D}+246,039$ & 0,96 & $\hat{\mathrm{Y}}=15,9061 \mathrm{D}+28,216$ & 0,95 \\
\hline 4,20 & $\hat{Y}=36,0078 D+256,208$ & 0,98 & $\hat{\mathrm{Y}}=15,3612 \mathrm{D}+35,931$ & 0,94 \\
\hline \multirow[t]{2}{*}{8,40} & $\hat{\mathrm{Y}}=31,8112 \mathrm{D}+273,109$ & 0,97 & $\hat{\mathrm{Y}}=17,7389 \mathrm{D}+24,206$ & 0,98 \\
\hline & \multicolumn{4}{|c|}{ Fluazifop-p-butil + Fomesafen } \\
\hline 0,00 & $\hat{\mathrm{Y}}=30,0924 \mathrm{D}+216,964$ & 0,93 & $\hat{\mathrm{Y}}=16,0272 \mathrm{D}+9,740$ & 0,95 \\
\hline 1,05 & $\hat{Y}=36,5020 D+162,393$ & 0,91 & $\hat{\mathrm{Y}}=17,2522 \mathrm{D}+5,586$ & 0,98 \\
\hline 2,10 & $\hat{\mathrm{Y}}=34,4824 \mathrm{D}+176,416$ & 0,93 & $\hat{\mathrm{Y}}=18,5120 \mathrm{D}+12,174$ & 0,98 \\
\hline 4,20 & $\hat{\mathrm{Y}}=33,4512 \mathrm{D}+191,898$ & 0,97 & $\hat{\mathrm{Y}}=18,0253 \mathrm{D}+35,091$ & 0,98 \\
\hline 8,40 & $\hat{\mathrm{Y}}=30,9017 \mathrm{D}+239,164$ & 0,89 & $\hat{\mathrm{Y}}=15,9844 \mathrm{D}+76,198$ & 0,94 \\
\hline
\end{tabular}

** Significativo pelo teste $\mathrm{t}$ a $1 \%$ de probabilidade de erro.

Tabela 3 - Carbono da biomassa microbiana (CBM) $\left(\mu \mathrm{g} \mathrm{g}^{-1}\right)$ em solo cultivado sob sistemas de plantio direto (SPD) e convencional (SPC) tratado com os herbicidas fluazifop-p-butil e fomesafen e a mistura comercial destes (Robust $\left.{ }^{\circledR}\right)$, com incubação ao longo de 20 dias. Viçosa-MG, 2005

\begin{tabular}{|c|c|c|c|c|c|c|}
\hline \multirow{2}{*}{$\begin{array}{c}\text { Concentração } \\
\left(\mu \mathrm{g} \mathrm{g}^{-1}\right)\end{array}$} & \multicolumn{6}{|c|}{ CBM $\left(\mu \mathrm{g} \mathrm{g}^{-1}\right)-\mathrm{SPD}$} \\
\hline & \multicolumn{2}{|c|}{ Fluazifop-p-butil } & \multicolumn{2}{|c|}{ Fomesafen } & \multicolumn{2}{|c|}{ Fluazifop-p-butil + Fomesafen } \\
\hline 0,00 & \multicolumn{6}{|c|}{582,63} \\
\hline 1,05 & \multicolumn{2}{|c|}{$521,05 \mathrm{a}$} & \multicolumn{2}{|c|}{$601,58 \mathrm{a}$} & \multicolumn{2}{|c|}{$544,74 \mathrm{a}$} \\
\hline 2,10 & \multicolumn{2}{|c|}{$445,26 \mathrm{~b}$} & \multicolumn{2}{|c|}{$630,00 \mathrm{a}$} & \multicolumn{2}{|c|}{$540,34 \mathrm{ab}$} \\
\hline 4,20 & \multicolumn{2}{|c|}{$492,63 \mathrm{~b}$} & \multicolumn{2}{|c|}{$634,74 \mathrm{a}$} & \multicolumn{2}{|c|}{$540,00 \mathrm{ab}$} \\
\hline 8,40 & \multicolumn{2}{|c|}{$596,84 \mathrm{a}$} & \multicolumn{2}{|c|}{$421,58 \mathrm{~b}$} & \multicolumn{2}{|c|}{$538,11 \mathrm{ab}$} \\
\hline $\mathrm{CV}(\%)$ & \multicolumn{6}{|c|}{14,19} \\
\hline \multirow[b]{2}{*}{$\begin{array}{c}\text { Concentração } \\
\left(\mu \mathrm{g} \mathrm{g}^{-1}\right)\end{array}$} & \multicolumn{3}{|c|}{$\operatorname{CBM}\left(\mu \mathrm{g} \mathrm{g}^{-1}\right)-\mathrm{SPC}$} & \multicolumn{3}{|c|}{ Quociente metabólico $\left(q \mathrm{CO}_{2}\right)-\mathrm{SPC}$} \\
\hline & $\begin{array}{l}\text { Fluazifop-p- } \\
\text { butil }\end{array}$ & Fomesafen & $\begin{array}{c}\text { Fluazifop-p- } \\
\text { butil + } \\
\text { Fomesafen }\end{array}$ & $\begin{array}{l}\text { Fluazifop-p- } \\
\text { butil }\end{array}$ & Fomesafen & $\begin{array}{c}\text { Fluazifop-p- } \\
\text { butil }+ \\
\text { Fomesafen }\end{array}$ \\
\hline 0,00 & \multicolumn{3}{|c|}{224,74} & \multicolumn{3}{|c|}{0,148} \\
\hline 1,05 & $154,09 \mathrm{a}$ & $222,78 \mathrm{a}$ & $157,96 \mathrm{a}$ & $1,99 \mathrm{a}$ & $1,52 \mathrm{a}$ & $2,67 \mathrm{a}$ \\
\hline 2,10 & $169,18 \mathrm{ab}$ & 226,89 a & $153,80 \mathrm{~b}$ & $2,09 \mathrm{a}$ & $1,62 \mathrm{a}$ & $2,28 \mathrm{a}$ \\
\hline 4,20 & $179,90 \mathrm{a}$ & $196,55 \mathrm{a}$ & $126,10 \mathrm{a}$ & $2,15 \mathrm{a}$ & $2,02 \mathrm{a}$ & $3,36 \mathrm{a}$ \\
\hline 8,40 & $186,04 \mathrm{a}$ & $114,84 \mathrm{ab}$ & $58,926 \mathrm{~b}$ & $2,21 \mathrm{a}$ & $2,95 \mathrm{a}$ & $7,28 \mathrm{~b}$ \\
\hline $\mathrm{CV}(\%)$ & \multicolumn{3}{|c|}{18,43} & \multicolumn{3}{|c|}{19,99} \\
\hline
\end{tabular}

Médias seguidas de letras iguais, na linha, não diferem entre si pelo teste de Tukey a 5\% de probabilidade. 
SPC, permitindo maior contato do herbicida com a comunidade microbiana presente. $\mathrm{O}$ distúrbio causado pelo revolvimento do solo impede a formação de microssítios propícios à atividade microbiana. Além disso, neste sistema de cultivo, as camadas subsuperficiais do solo são constantemente revolvidas, expondo a microbiota às intempéries climáticas. Especificamente no caso da mistura comercial dos herbicidas, o efeito tóxico sobre a microbiota pode ter ocorrido pela ação conjunta dos herbicidas, isto é, dois mecanismos diferentes atuando ao mesmo tempo. O fluazifop-p-butil, apesar de ter sido menos tóxico à microbiota no SPD e no SPC quando aplicado isoladamente, é um potente inibidor da síntese de acetil coenzima A carboxilase (ACCase) (Hess, 1995), presente também no metabolismo microbiano.

Entre os herbicidas, nas diferentes concentrações, verificou-se que, em SPD, a mistura comercial não promoveu variação no CBM. Contudo, considerando cada herbicida isoladamente, o fluazifop-p-butil provocou diminuição na biomassa microbiana até a concentração de $3,57 \mu \mathrm{g} \mathrm{g}^{-1}$ e aumento de CBM a partir desse valor, enquanto para fomesafen o comportamento foi contrário, com diminuição no $\mathrm{CBM}$ nas maiores concentrações (Figura 3). A retomada no aumento de CBM nas maiores concentrações do fluazifop-p-butil pode ser explicada pela presença de microrganismos saprofíticos de crescimento rápido, os quais se beneficiam da matéria orgânica advinda da morte da comunidade anteriormente presente. $\mathrm{O}$ mesmo não foi observado para o fomesafen, talvez por sua maior agressividade aos microrganismos, já comentada, associado ao maior efeito residual desse produto em relação ao fluazifop-p-butil.

Em solo proveniente do SPD, os valores de CBM diminuíram mais expressivamente para a mistura dos herbicidas. Apesar de a biomassa microbiana apresentar pouca variação entre as concentrações de fluazifop-p-butil, concluiu-se que este herbicida tem efeito inibitório sobre a biomassa microbiana, pois a menor concentração adicionada ao solo $\left(1,05 \mu \mathrm{g} \mathrm{g}^{-1}\right)$ foi suficiente para causar decréscimo de $11 \%$ no CBM. Ao contrário, o fomesafen apresentou-se menos tóxico nas menores concentrações, porém promoveu redução do CBM a valores abaixo dos observados para fluazifop-p-butil em maiores concentrações no solo (Figura 3).

A relação entre o $\mathrm{CO}_{2}$ acumulado e o total do CBM proporciona o denominado quociente metabólico $\left(\mathrm{qCO}_{2}\right)$ proposto por Anderson \& Domsch (1985) e prediz que, à medida que a biomassa microbiana se torna mais eficiente em utilizar os recursos em seu meio, menos $\mathrm{C}$ é perdido como $\mathrm{CO}_{2}$ pela respiração, podendo este ser incorporado aos tecidos microbianos. Para Sakamoto \& Obo (1994), solos com alto $\mathrm{qCO}_{2}$ são dominados por organismos colonizadores de crescimento rápido, refletindo um ambiente mais instável ou mais longe de seu estado de equilíbrio. Dessa forma, menor $\mathrm{qCO}_{2}$
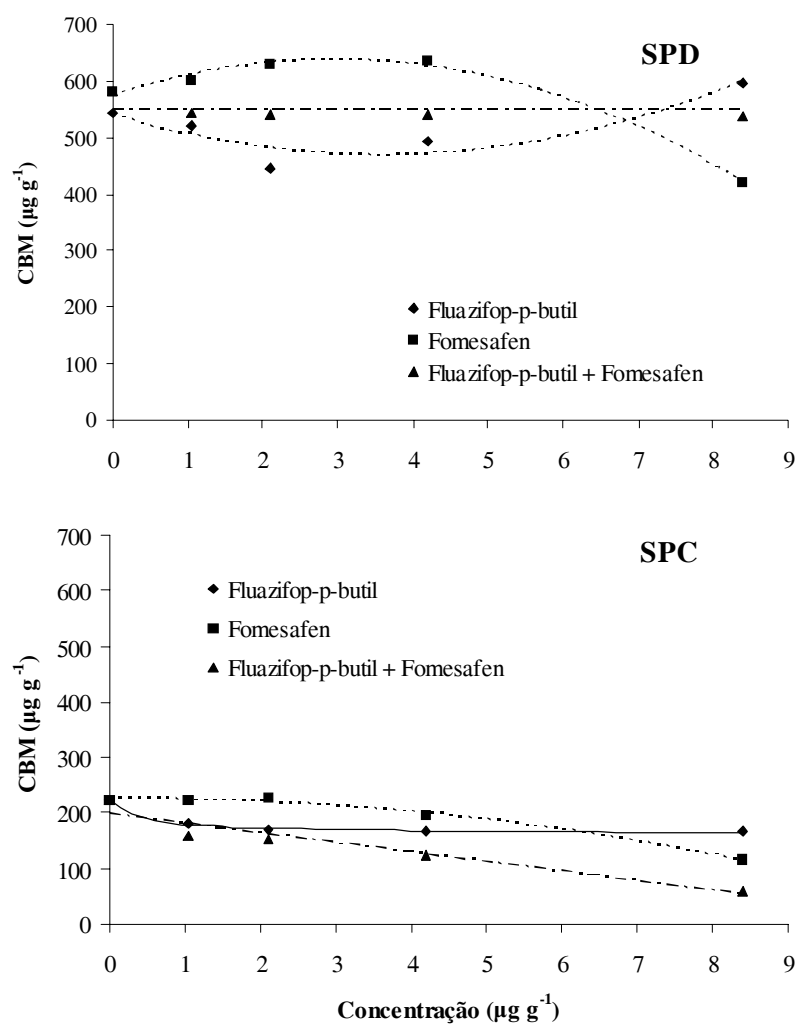

Figura 3 - Carbono da biomassa microbiana (CBM) determinado em solo sob diferentes concentrações (C) dos herbicidas fluazifop-p-butil (Sistema de Plantio Direto SPD: $\hat{Y}=5,5919 C^{2}-39,9810 C+541,241 R^{2}=0,84$; e Sistema de Plantio Convencional - SPC: $\hat{Y}=162,4306+(24,0372) /$ $\left.0,3854+C) R^{2}=0,99\right)$ e fomesafen (SPD: $\hat{Y}=7,3082 C^{2}+$ $43,3451 C+574,539 R^{2}=0,99$ e SPC: $\hat{Y}=-1,7216 C^{2}+$ $\left.1,2016 C+225,639 R^{2}=0,98\right)$ e da mistura comercial destes (Robust $\left.{ }^{\circledR}\right)$ (SPD: $\bar{Y}=549,189 I$ e SPC: $\hat{Y}=-17,219 C+$ $\left.198,551 R^{2}=0,91\right)$.

Planta Daninha, Viçosa-MG, v. 23, n. 4, p. 683-691, 2005 
significa maior estabilidade da biomassa microbiana. No solo provindo do SPD não se observaram diferenças no $\mathrm{qCO}_{2}$ entre os herbicidas (Tabela 3). No entanto, com o aumento das concentrações, verificou-se incremento do $\mathrm{qCO}_{2}$, indicando que a biomassa microbiana se torna menos eficiente na presença dos herbicidas, sendo essa eficiência tão menor quanto maior a concentração destes (Figura 4).

A mesma avaliação realizada para o mesmo solo sob SPC revelou diferenças de $\mathrm{qCO}_{2}$ entre os herbicidas. Enquanto para os herbicidas isolados, na maior concentração testada, o valor médio para $\mathrm{qCO}_{2}$ foi de 2,58 , a mistura
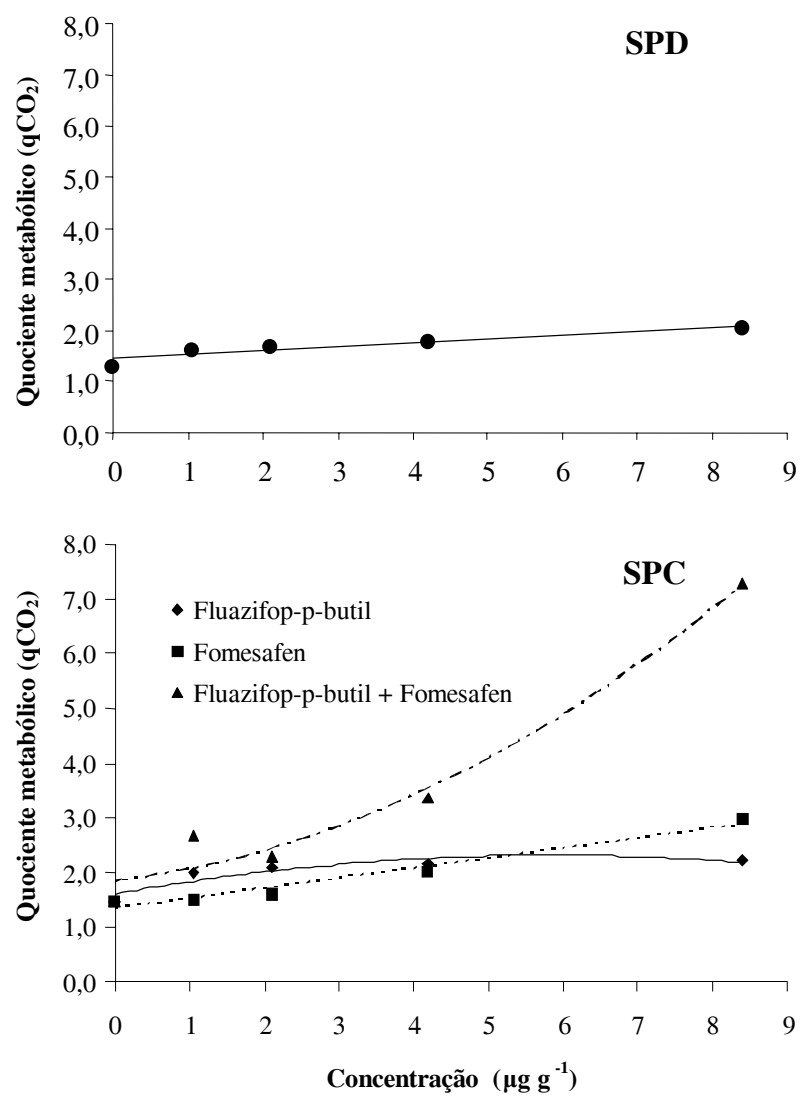

Figura 4 - Quociente metabólico $\left(\mathrm{qCO}_{2}\right)$ médio determinado em solo, proveniente de sistema de plantio direto (SPD), sob diferentes concentrações (C) $(\hat{Y}=0,0757 C+1,454$ $\left.R^{2}=0,87\right)$ dos herbicidas fluazifop-p-butil e fomesafen e da mistura comercial destes (Robust ${ }^{\circledR}$ ) e de sistema de plantio convencional (SPC), sob ação do fluazifop-p-butil $\left(\hat{Y}=0,0208 C^{2}+0,243 C+1,6092 R^{2}=0,82\right)$, fomesafen $\left(\hat{Y}=0,1833 C+1,3407 R^{2}=0,96\right)$ e da mistura comercial destes (Robust $\left.{ }^{\circledR}\right)\left(\hat{Y}=0,0574 C^{2}+0,1623 C+1,8313\right.$ $R^{2}=0,94$ ). comercial desses produtos, na mesma concentração, elevou esse valor para 7,28 (Tabela 3). Com o aumento das concentrações de cada herbicida, o $\mathrm{qCO}_{2}$ sob influência de fluazifop-pbutil aumentou linearmente. Efeito semelhante, porém não linear, foi observado quando o SPC foi tratado com o fomesafen. Este efeito foi ainda mais expressivo na presença da mistura comercial de fluazifop-p-butil + fomesafen (Robust ${ }^{\circledR}$ ) (Figura 4). Isso pode indicar que os herbicidas testados, por apresentarem diferentes mecanismos de ação, quando aplicados isoladamente, estariam afetando uma parcela da comunidade microbiana sensivel a cada produto. Contudo, a aplicação da mistura de ambos potencializa o efeito inibitório sobre a atividade microbiana, promovendo maior instabilidade nos processos microbiológicos $\mathrm{e}$ bioquímicos do solo, representados pelo $\mathrm{qCO}_{2}$.

Na ausência de herbicidas, a avaliação do $\mathrm{qCO}_{2}$ indica a estabilidade entre os sistemas de cultivo. Considerando que o valor de $\mathrm{qCO}_{2}$ observado em solo do SPC foi $13 \%$ superior ao observado no SPD, fica evidenciada a maior estabilidade deste sistema de cultivo sobre o SPC. Da mesma forma, a aplicação dos diferentes herbicidas, nas concentrações testadas, promoveu maior aumento no $\mathrm{qCO}_{2}$ quando no SPC, ou seja, o impacto negativo da aplicação desses herbicidas sobre a microbiota do solo é diminuído no SPD.

\section{AGRADECIMENTO}

Ao CNPq pelo apoio financeiro.

\section{LITERATURA CITADA}

AMATO, M.; LADD, J. N. Decomposition of 14Clabelled glucose and legume material in soil: Properties influencing the accumulation of organic residue $\mathrm{C}$ and microbial biomass C. Soil Biol. Biochem., v. 24, p. 455-464, 1992.

ANDERSON, T. H.; DOMSCH, K. H. Determination of ecophysiological maintenance carbon requirements of soil microorganisms in a dormant state. Biol. Fertil. Soils, v. 1, p. 81-89, 1985.

BALOTA, E. L. et al. Microbial biomass in soils under different tillage and crop rotation systems. Biol. Fertil. Soil, v. 38, p. 15-20, 2003.

BEARE, M. H. et al. A hierarchical approach to evaluating the significance of soil biodiversity to biogeochemical cycling. Plant Soil, v. 170, p. 5-22, 1995. 
CARTER, M. R. Influence of reduced tillage systems on organic matter, microbial biomass, macro-aggregate distribution and structural stability of surface soil in a humid climate. Soil Till. Res., v. 23, p. 361-372, 1992.

EDWARDS, C. A. Impact of herbicides on soil ecosystems. Crit. Rev. Plant Sci., v. 8, p. 221-257, 1989.

ELLIOTT, E. T.; COLEMAN, D. C. Let the soil work for us. Ecol. Bull., v. 39, p. 23-32, 1988.

EMPRESA BRASILEIRA DE PESQUISA AGROPECUÁRIA - EMBRAPA. Centro Nacional de Pesquisa de Solos. Manual de métodos de análise de solo. 2.ed. Rio de Janeiro: 1997. 212 p.

FEDERAÇÃO BRASILEIRA DE PLANTIO DIRETO NA PALHA - FEBRAPDP. http://www.febrapdp.org.br./ area_PD_Brasil_(12 mar. 2005).

FONTES, J. R. A. et al. Lixiviação de herbicidas no solo aplicados com água de irrigação em plantio direto. Planta Daninha, v. 22, p. 623-631, 2004.

HESS, F. D. Mode of action of lipid biosyntesis inhibitors (Graminicides - Accase Inhibitors). In: Herbicide action course. West Laffeyte: Purdue University, 1995. 787 p.

ISLAM, K. R.; WEIL, R. R. Microwave irradiation of soil for routine measurement of microbial biomass carbon. Biol. Fertil. Soils, v. 27, p. 408-416, 1998.

JENKINSON, D. S.; LADD, J. M. Microbial biomass in soil: measurement and turnover. In: PAUL, E. A.; LADD, J. N. (Eds.) Soil biochemistry. New York: Marcel Dekker, 1981. v. 5, p. 415-471.

KINNEY, C. A.; MANDERNACK, K. W.; MOSIER, A. R. Laboratory investigations into the effects of the pesticides mancozeb, chlorothalonil, and prosulfuron on nitrous oxide and nitric oxide production in fertilized soil. Soil Biol. Biochem., v. 37, p. 837-850, 2005.

PERSCHBACHER, P. W. et al. Evaluation of effects of common aerially- applied soybean herbicides and propanyl on the plankton communities of aquaculture ponds. Aquaculture, v. 157, p. 117-122, 1997.

PROCÓPIO, S. O. et al. Crescimento de estirpes de Bradyrhizobium sob influência dos herbicidas glyphosate potássico, fomesafen, imazethapyr e carfentrazone-ethyl. R. Ceres, v. 51, n. 294, p. 179-188, 2004.
RODRIGUES, B. N.; ALMEIDA, F. S. Guia de herbicidas. 4.ed. Londrina: 1998. 648 p.

SAKAMOTO, K.; OBO, Y. Effects of fungal to bacterial ratio on the relationship between $\mathrm{CO} 2$ evolution and total soil microbial biomass. Biol. Fertil. Soils, v. 17, p. 39-44, 1994.

SANTOS, J. B. Efeitos do glyphosate sobre o crescimento de estirpes de Bradyrhizobium. 2004. $56 \mathrm{f}$. Dissertação (Mestrado em Fitotecnia) - Universidade Federal de Viçosa, Viçosa-MG, 2004.

SILVA, A. A. et al. Controle de plantas daninhas. Brasília: ABEAS, 2003. 260 p.

SOUZA, A. P. et al. Respiração microbiana do solo sob doses de glyphosate e de imazapyr. Planta Daninha, v. 17, p. 387-398, 1999.

SPARLING, G. P. Ratio of microbial biomass carbon to soil organic carbon as a sensitive indication of changes in soil organic matter. Aust. J. Soil. Res., v. 30, p. 195-207, 1992.

STENBERG, B. Monitoring soil quality of arable land: microbiological indicators. Soil Plant Sci., v. 49, p. 1-24, 1999.

TUFFI SANTOS, L.D. et al. Exsudação radicular do glyphosate por Brachiaria decumbens e seus efeitos em plantas de eucalipto e na respiração microbiana do solo. Planta Daninha, v. 23, p. 143-152, 2005.

VANCE, E. D.; BROOKES, P. C.; JENKINSON, D. S. An extraction method for measuring soil microbial biomass $\mathrm{C}$. Soil Biol. Biochem., v. 19, p. 703-707, 1987.

WARDLE, D. A. Impact of disturbances on detritus foodwebs in agro-ecosystems of contrasting tillage and weed management practices. Adv. Ecol. Res., v. 26, p. 10-17, 1994.

WARDLE, D. A.; PARKINSON, D. Relative importance of the effects of 2,4-D, glyphosate and environmental variables on the soil microbial biomass. Plant Soil, v. 134, p. 209219, 1991.

WARREN, G. F.; HESS, F. D. Mode of action of inhibitors of protoporphyrinogen oxidase (Diphenilethers and oxadiazon). In: Herbicide action course. West Laffeyte: Purdue University, 1995. 787 p. 